\title{
Economics and safety of continuous and interrupted suture hepaticojejunostomy: An audit of 556 surgeries
}

\author{
Rajan Saxena, Nalini Kanta Ghosh, Saurabh Galodha \\ Department of Surgical Gastroenterology and Center of Hepatobiliary Diseases and Transplantation, \\ Sanjay Gandhi Post Graduate Institute of Medical Sciences, Lucknow, India
}

Backgrounds/Aims: Hepaticojejunostomy $(\mathrm{HJ})$ for bilioenteric continuity is generally performed with interrupted sutures. This study compares the safety, economics, short- and long-term outcomes of continuous suture hepaticojejunostomy (CSHJ) and interrupted suture hepaticojejunostomy (ISHJ).

Methods: A retrospective cohort analysis involving all HJs between January 2014 and December 2018 was conducted. Patients with type IV or V biliary strictures, duct diameter $<8 \mathrm{~mm}$ and/or associated vascular injury, and liver transplant recipients were excluded. Patient demographics, preoperative parameters including diagnosis, intra-operative parameters including type and number of sutures, suture time, and postoperative morbidity (based on Clavien-Dindo classification) were recorded. Patients were followed up to 60 months. McDonald's Grade A and B outcomes were considered favorable. Cost according to suture type and number (polydioxanone 3-0/5-0 mean cost, US\$ 9.26/length; polyglactin 3-0/4-0 mean cost, US\$ 6.56/length), and operation room charge (US\$ 67.47/hour) were compared between the two techniques. Statistical analysis was performed using IBM SPSS ver. 22 software.

Results: A total of 556 eligible patients (468 patients undergoing ISHJ and 88 undergoing CSHJ; 47\% [ $\mathrm{n}=261$ ] with malignant and $53 \%$ [ $=295$ ] with benign pathology) were analyzed. The two groups were similar. Number of sutures, cost, time, and postoperative bile leak were significantly higher in the ISHJ group. Bile leak occurred in 54 patients (6 CSHJ, 48 ISHJ). Septic shock-induced death occurred in 16 cases (3 CSHJ, 13 ISHJ). Morbidity and the anastomotic stricture rates were comparable in both groups.

Conclusions: $\mathrm{CSHJ}$ is a safe, economical, and worthy of routine use.

Key Words: Continous suture hepaticojejunostomy; Interrupted suture hepaticojejunostomy; Cost of hepaticojejunostomy, economics, anastomotic time; Hepaticojejunostomy outcome

\section{INTRODUCTION}

Hepaticojejunostomy $(\mathrm{HJ})$ is a common and standardized method of bilio-enteric reconstruction, performed either as primary surgery or as part of a major surgical procedure

Received: June 2, 2021, Revised: July 10, 2021,

Accepted: July 26, 2021

Corresponding author: Rajan Saxena

Department of Surgical Gastroenterology and Center of Hepatobiliary Diseases and Transplantation, Sanjay Gandhi Post Graduate Institute of Medical Sciences, Raebareli Road, Lucknow 226014, India

Tel: +91-522-2494425/2494403, Fax: +91-522-2668017,

E-mail: rajansaxena1959@gmail.com

ORCID: https://orcid.org/0000-0002-3122-0140

Copyright (C) The Korean Association of Hepato-Biliary-Pancreatic Surgery

This is an Open Access article distributed under the terms of the Creative Commons Attribution Non-Commercial License (http://creativecommons.org/licenses/by-nc/4.0) which non-commercial use, distribution, and reproduction in any medium, provided the original permits unrestricted not work is properly cited. such as pancreaticoduodenectomy, choledochal cyst excision and hepatectomy with extrahepatic biliary tree excision. The anastomosis can be performed either as a continuous suture hepaticojejunostomy ( $\mathrm{CSHJ})$ or as an interrupted suture hepaticojejunostomy (ISHJ). Most surgeons prefer ISHJ. CSHJ is a quicker method but associated with the risk of anastomotic site strictures. The CSHJ technique is comparable to ISHJ technique in liver transplantation in terms of biliary complications [1-4]. The economics and safety of CSHJ and ISHJ in terms of complications (early and late) and related patient costs as well as the difference in operative time have yet to be reported. This study compares the safety and economic implications (material cost and time) associated with the two methods of anastomosis as well as the postoperative course and long-term outcomes in non-transplant patients. 


\section{MATERIALS AND METHODS}

This retrospective observational cohort study includes all consecutive patients undergoing bilio-enteric anastomosis in the form of HJ between January 2014 and December 2018 at a tertiary care center in north India.

\section{Inclusion criteria}

The study includes patients with bile duct diameter greater than 8-mm undergoing $\mathrm{HJ}$ irrespective of the pathology. As the space available for needle movement in CSHJ is progressively reduced in a small or normal duct, ducts smaller than $8 \mathrm{~mm}$ are reconstructed with interrupted sutures routinely at our center. Prior sutures provide adequate room for maneuver.

\section{Exclusion criteria}

Patients with types IV and V biliary strictures, carrying more than one bilio-enteric anastomosis, those with concomitant vascular injury (hepatic artery or portal vein) during dissection, and patients undergoing $\mathrm{HJ}$ as biliary reconstruction during liver transplantation were excluded.

Preoperative demographic profiles and primary pathologies were recorded. Intra-operative parameters were recorded, together with operative findings, including the number and type of sutures, and the complete anastomotic time. The anastomotic time was determined from taking the first suture bite until the tying of the last knot. Polyglactin 3-0 and 4-0 (cost US\$ 6.56 per suture) and Polydioxanone (PDS) 3-0, 4-0, and 5-0 sutures (cost US\$ 9.26 per suture length) (ETHICON, Aurangabad, India) were used for $\mathrm{HJ}$. Operating room charges were billed at the rate of US\$ 67.47 per hour. These cost factors were compared between the two methods. Early post-operative course of patients was diligently recorded including the nature and volume of drain output, the day of drain removal, complications in the form of bleed, bile leak, sepsis, type of intervention and re-exploration if required. Morbidity was graded according to Clavien-Dindo classification [5]. Suspected HJ leak was established via cross-sectional imaging using contrast-enhanced computed tomography (CT) scan of the abdomen and guided aspiration of the collection. Interventions included single-time aspiration or percutaneous drainage (PCD) under ultrasound or CT guidance. The abdomen was re-explored in patients with hemodynamic instability or uncontrolled sepsis. HJ outcomes were reported using McDonald's criteria [6] in the surviving 540 patients. Grade A and B findings were regarded as good outcomes. The patients were followed up at regular intervals post-operatively (initially at 3 months, 6 months and then yearly) with abdominal sonography and liver function tests. Magnetic resonance cholangiopancreatography was performed in case of increased levels of bilirubin, alkaline phosphatase or cholangitis, and further management was based on clinical and radiological parameters. All data were retrieved from follow-up records.

\section{Statistical analysis}

All parameters were recorded in an Excel sheet (Microsoft Excel 2016; Microsoft, Redmond, WA, USA). Continuous variables were expressed as means and standard deviations. In case of skewed variables medians and interquartile ranges (IQRs) were used. Continuous variables were compared using the Wilcoxon test, whereas categorical variables were compared using Fisher's exact test or chi-square test. A $p$-value $<0.05$ was considered significant. All statistical analyses were done using Statistical Package for the Social Sciences software (IBM SPSS Statistics ver. 22; IBM Corp., Armonk, NY, USA).

\section{RESULTS}

HJ was performed in 586 patients during the study period. Thirty patients were excluded based on exclusion criteria. The study included a total of 556 eligible patients finally. Demographic and clinical characteristics of both groups (continuous

Table 1. Demographic and clinical characteristics of patients undergoing $\mathrm{CSHJ}$ and ISHJ

\begin{tabular}{|c|c|c|c|}
\hline Parameter & $\mathrm{CSHJ}(\mathrm{n}=88)$ & $\mathrm{ISHJ}(\mathrm{n}=468)$ & $p$-value \\
\hline Age in years & $48.7 \pm 16.4$ & $46.2 \pm 14.01$ & 0.13 \\
\hline $\mathrm{BMI}\left(\mathrm{kg} / \mathrm{m}^{2}\right)$ & $21.7 \pm 3.05$ & $21.4 \pm 3.06$ & 0.34 \\
\hline Sex (male/female) & $46.6 \% / 53.4 \%$ & $40.0 \% / 60.0 \%$ & 0.14 \\
\hline Benign etiology & $46(52.3)$ & $249(53.2)$ & 0.4 \\
\hline CDC & $20(22.7)$ & $92(19.6)$ & 0.29 \\
\hline BBS & $21(23.9)$ & $134(28.6)$ & 0.22 \\
\hline Choledocholithiasis & $1(1.1)$ & $10(2.1)$ & 0.46 \\
\hline Mirizzi syndrome & $2(2.3)$ & $7(1.5)$ & 0.43 \\
\hline Others & $2(2.3)$ & $6(1.3)$ & 0.69 \\
\hline Malignant & $42(47.2)$ & $219(46.8)$ & 0.4 \\
\hline Periampullary carcinoma & $26(29.5)$ & $165(35.2)$ & 0.18 \\
\hline Ca HOP & $6(6.8)$ & $12(2.6)$ & 0.05 \\
\hline CAGB & $3(3.4)$ & $7(1.5)$ & 0.2 \\
\hline DCCA & $4(4.5)$ & $22(4.7)$ & 0.6 \\
\hline Others & $3(3.4)$ & $13(2.8)$ & 0.47 \\
\hline \multicolumn{4}{|l|}{ Intra-operative parameter } \\
\hline Type of suture (Vicryl) & $24(27.2)$ & $363(77.5)$ & $<0.001$ \\
\hline Number of sutures & $2.16 \pm 0.43$ & $13.04 \pm 4.05$ & $<0.001$ \\
\hline Duct diameter (mm) & $17(8-90)$ & $15(8-100)$ & 0.46 \\
\hline Stoma size $(\mathrm{mm})$ & $20.4 \pm 9.5$ & $20.2 \pm 7.5$ & 0.6 \\
\hline $\begin{array}{l}\text { Duration of anastomosis } \\
\text { (min) }\end{array}$ & $21.4 \pm 11.2$ & $44.6 \pm 20.3$ & $<0.001$ \\
\hline Cost in US\$ & $17.42 \pm 2.91$ & $104.18 \pm 26.08$ & $<0.001$ \\
\hline Duration of drain in days & $6(1-28)$ & $5(2-29)$ & 0.91 \\
\hline Hospital stays in days & $9(5-60)$ & $10(2-65)$ & 0.47 \\
\hline \multicolumn{4}{|c|}{$\begin{array}{l}\text { Values are presented as mean } \pm \text { standard deviation, number }(\%) \text {, or } \\
\text { median (inter quartile range). }\end{array}$} \\
\hline
\end{tabular}


and interrupted anastomosis) are listed in Table 1. Age, gender distribution and pathology were comparable between the two groups. Ten consultant surgeons and 20 trainee residents participated in this study. All CSHJs and 46 ISHJs were carried out by the senior author, who is a liver transplant surgeon. All other HJs were performed by surgical gastroenterology consultants as well as trainee residents who performed $80 \%$ of the procedures under supervision.

The patient population $(n=556)$ manifested benign $(n=295$; $53 \%)$ and malignant conditions $(\mathrm{n}=261 ; 47 \%)$. The distribution of pathology was comparable in the two groups as shown in Table 1.

All patients included in this study had a ductal diameter of 8 mm or more.

Intraoperative, Polyglactin 3-0 and 4-0 sutures and PDS 3-0, 4-0, and 5-0 sutures were used in HJ. Polyglactin was more frequently used in ISHJ than in CSHJ group (363/468 [77.5\%] vs. $24 / 88$ [27.2\%]; $p<0.001$ ) (Table 1). Suture lengths required to complete the anastomoses were greater in ISHJ group than in CSHJ group (13.04 vs. 2.16; $p<0.001$ ). Time taken to complete the anastomosis was less in CSHJ group than in ISHJ group $(21.4 \pm 11.2$ vs. $44.6 \pm 20.3$ minutes; $p<0.001)$. Cost of anastomosis was also less in CSHJ group than in ISHJ group US\$ $17.42 \pm 2.91$ vs. US $\$ 104.18 \pm 26.08 ; p<0.001$ ).

Bile leak following HJ was significantly higher in ISHJ group than in CSHJ group (48/468 [10.2\%] vs. 6/88 [6.8\%]; $p=0.04$ ) (Table 2), and did not correlate with the ductal diameter. Post-operative morbidities were comparable among both groups in terms of Clavien-Dindo grading. Grade IIIa morbidity was observed in 40 patients (CSHJ, 10; ISHJ, 30). Sixteen patients required PCD insertion (CSHJ, 3; ISHJ, 13) and 8 patients underwent single-time aspiration, and the remaining 16 experienced SSI requiring delayed skin suturing. Among 19 patients with grade IIIb morbidity (CSHJ, 3; ISHJ, 16), 2 patients in the ISHJ group required re-exploration for bile leak, and one patient required reinforcing sutures at $\mathrm{HJ}$ site and the other was managed with lavage and drainage to create a controlled external biliary fistula. Seventeen patients (89.5\%) required re-exploration for intra-abdominal bleeding unrelated to biliary reconstruction. One patient in the ISHJ group recovered following to intensive care unit support for managing sepsis and organ failure (Grade IV). Sixteen patients (2.8\%) showed 30-day mortality following surgery (3 [3.4\%] in the CSHJ compared with 13 [2.7\%] in the ISHJ group) due to sepsis, which was not statistically significant $(p=0.42)$. These 16 patients were excluded from the long-term outcome analysis for which 540 surviving patients were eligible.

Median postoperative hospital stay in both groups was comparable ( 9 vs. 10 days; $p=0.47$ ).

The median follow-up in all surviving patients was 24 months (range, 4-60 months). Long-term results of $\mathrm{HJ}$ were assessed using McDonald's criteria. Grade A and B findings were regarded as good outcome. Grade A outcomes were higher in
Table 2. Postoperative outcomes

\begin{tabular}{lccc}
\hline \multicolumn{1}{c}{ Parameter } & $\begin{array}{c}\text { CSHJ } \\
(\mathrm{n}=88)\end{array}$ & $\begin{array}{c}\text { ISHJ } \\
(\mathrm{n}=468)\end{array}$ & $p$-value \\
\hline Morbidity & $34(38.6)$ & $200(42.7)$ & 0.47 \\
Bile & $6(6.8)$ & $48(10.2)$ & $0.04^{*}$ \\
Clavien-Dindo Grade & & & \\
Grade 0 & $56(63.6)$ & $308(65.8)$ & 0.07 \\
Grade I & $14(15.9)$ & $88(18.8)$ & 0.2 \\
Grade II & $2(2.3)$ & $12(2.6)$ & 0.62 \\
Grade IIla & $10(11.4)$ & $30(6.4)$ & 0.17 \\
Grade IIIb & $3(3.4)$ & $16(3.4)$ & 0.07 \\
Grade IVa & 0 & $1(0.2)$ & 0.06 \\
Grade V & $3(3.4)$ & $13(2.7)$ & 0.42 \\
Follow up in months & $36(4-60)$ & $24(4-60)$ & 0.12 \\
McDonald's Grade (assessed in 540 surviving patients) & \\
No. of patients (total = 540) & 85 & 455 & \\
Grade A & $80(94.1)$ & $398(87.4)$ & 0.14 \\
Grade B & $1(1.2)$ & $28(6.1)$ & $0.04^{*}$ \\
Grade C & $0(0)$ & $8(1.7)$ & 0.25 \\
Grade D & $4(4.7)$ & $21(4.6)$ & 0.57 \\
\hline
\end{tabular}

Values are presented as number (\%) or median (range).

$\mathrm{CSHJ}$, continuous suture hepaticojejunostomy; ISHJ, interrupted suture hepaticojejunostomy.

*Statistically significant $(p<0.05)$.

the CSHJ group than in the ISHJ group, but the difference was not statistically significant $(p=0.14)$. The ISHJ group showed significantly higher grade B outcomes $(p=0.04)$. No grade C outcome was observed in the CSHJ group, whereas 8 patients in the ISHJ group showed grade $\mathrm{C}$ outcomes $(p=0.25)$. Twenty-five of the 540 surviving patients analyzed (4.6\%) developed $\mathrm{HJ}$ anastomotic stricture requiring intervention (4 [4.7\%] in the CSHJ group and 21 [4.6\%] in the ISHJ group), and the difference was not statistically significant $(p=0.57)$.

\section{DISCUSSION}

$\mathrm{HJ}$ is an accepted method of bilio-enteric reconstruction in many benign and malignant conditions. However, an anastomotic leak or stricture can lead to significant morbidity and mortality [7]. Among the methods of anastomosis, interrupted suturing is preferred by most surgeons due to the notion that ISHJ reduces the incidence of anastomotic site stricture, although it is not supported by evidence. Evaluation of liver transplant recipients showed that CSHJ was equally efficacious without an increased incidence of anastomotic strictures (Table 3); however, it has yet to be validated in the non-transplant population.

Studies comparing the safety and economic value of CSHJ and ISHJ groups are scarce. Galodha and Saxena [8] prospectively compared the cost of sutures (number and length) and operation room time associated with CSHJ and ISHJ in 40 pa- 
Table 3. Studies comparing CSHJ with ISHJ

\begin{tabular}{|c|c|c|c|c|c|c|}
\hline Author & Year & Population & $\begin{array}{l}\text { No. of } \\
\text { CSHJ/ISHJ }\end{array}$ & $\begin{array}{l}\text { Follow } \\
\text { up (mon) }\end{array}$ & Outcome & $p$-value \\
\hline Kasahara et al. [1] & 2006 & Liver transplant & $48 / 68$ & 60 & $\begin{array}{l}\text { - Bile leak (CSHJ, 8.3\%; ISHJ, 14.7\%) } \\
\text { - Stricture (CSHJ, 5\%; ISHJ, 5\%) }\end{array}$ & $>0.05$ \\
\hline Soejima et al. [2] & 2006 & Liver transplantation & $5 / 53$ & 36 & • Stricture (CSHJ, 0\%; ISHJ, 31.8\%) & \\
\hline Castaldo et al. [3] & 2007 & Liver transplant & $41 / 59$ & $\begin{array}{l}15 \pm 7 \\
17 \pm 8\end{array}$ & $\begin{array}{l}\text { - Bile leak (CSHJ, 7.3\%, ISHJ, 8.5\%) } \\
\text { - Stricture (CSHJ, 9.8\%, ISHJ, 5.1\%) }\end{array}$ & $\begin{array}{l}0.83 \\
0.37\end{array}$ \\
\hline Galodha and Saxena [8] & 2016 & Non transplant & $40 / 40$ & - & $\begin{array}{l}\text { - Duration }(\mathrm{min})(\mathrm{CSHJ}, 16.7 \pm 4.7 ; \mathrm{ISHJ}, 46.8 \pm 17.5) \\
\text { - No. of sutures (CSHJ, 2; ISHJ, 15) } \\
\text { - Bile leak (CSHJ, 1; ISHJ, 4) }\end{array}$ & $<0.01$ \\
\hline Abdel Hamid et al. [13] & 2020 & Non transplant & $20 / 23$ & - & $\begin{array}{l}\text { - Duration }(\mathrm{min})(\mathrm{CSHJ}, 18.85 \pm 3.2, \mathrm{ISHJ}, 27.56 \pm 3.7) \\
\text { - No. of sutures }(\mathrm{CSHJ}, 2 ; \mathrm{ISHJ}, 10)\end{array}$ & $<0.01$ \\
\hline
\end{tabular}

Values are presented as mean \pm standard deviation or range.

$\mathrm{CSHJ}$, continuous suture hepaticojejunostomy; ISHJ, interrupted suture hepaticojejunostomy.

tients under each group. The findings suggested that CSHJ was economical and safe (Table 3). Castaldo et al. [3] compared the advantages of these methods in biliary reconstruction during liver transplantation. The anastomotic time did not differ; however, in our study it was substantially reduced in CSHJ than in ISHJ group $(p<0.01)$. The number of sutures used and the cost of anastomosis were lower in CSHJ than in ISHJ group in Castaldo's study, consistent with our observations. In Castaldo's report [3], the bile leak rate in CSHJ and ISHJ groups was $7.3 \%$ and $8.5 \%$, respectively, with a $p$-value of 0.83 . Our study showed bile leak rates of $6.8 \%$ and $10.2 \%$, which was statistically significant $(p=0.04)$. The higher leak rate may be associated with the training of the surgeon executing the step; however, the stricture rate in their study was not significantly different $(p=0.37)$. Stricture rate in our study was lower than in their study. Tsui et al. [4] reported a $6.7 \%$ incidence of anastomotic strictures following continuous suture end-to-end biliary anastomosis, but in a different subset of liver transplant recipients.

The overall bile leak rate following $\mathrm{HJ}$ varies from $2.3 \%$ to $5.6 \%$, irrespective of the type of anastomosis [7,9]. In our study population, the incidence of bile leak was higher (CSHJ, 6.8\%; ISHJ $10.2 \%$ ) and was significantly higher in the ISHJ group. The incidence of anastomotic strictures reported in the literature is $3.7 \%-8.0 \%[10,11]$. The overall stricture rate of $4.6 \%$ in the present study is comparable. The stricture rate was higher in benign pathologies when compared with malignant diseases, possibly due to differences in survival and the resultant follow-up time. Table 3 presents a comparison of different studies reporting the two techniques of anastomosis.

A nationwide survey in Germany by Brunner et al. [12], covering 77 hospitals also concluded that CSHJ was significantly quicker than ISHJ ( $p=0.015)$, without significant difference in leak rate and overall morbidity, consistent with our observations, although long-term outcomes have yet to be reported. Abdel Hamid et al. [13] also reported that the number of sutures and the duration of anastomosis were significantly less in $\mathrm{CSHJ}$ group, but the long-term outcomes were unavailable.

In the present study, Polyglactin suture was more commonly used in ISHJ while PDS was preferred for the CSHJ group. The number of sutures required, the anastomotic time and the overall cost of anastomosis were significantly less in the CSHJ group than in the ISHJ group. The incidence of HJ leak was significantly higher in the ISHJ group when compared with the $\mathrm{CSHJ}$ group, and was more pronounced in benign pathology. Most of the re-explorations were done for intra-abdominal bleeding. The overall incidence of biliary stricture and median hospital stay amongst both groups were comparable. There were 16 mortalities due to sepsis (CSHJ: 3, ISHJ: 13; malignant etiology: 15 , benign etiology: 1$)$, a majority of which $(15 / 16$; 93.8\%) occurred in the malignant group and were multifactorial in etiology. McDonald Grade-D (anastomotic site stricture requiring intervention) was also comparable amongst both the groups, although Soejima et al. [2] reported a zero incidence of anastomotic strictures in 5 patients undergoing CSHJ for living donor liver transplant (LDLT) after a 36-month follow-up period, compared with a $31.8 \%$ stricture rate following ISHJ in 53 
patients undergoing LDLT after similar follow-up.

The limitation of this study is that patients undergoing liver transplant were excluded from the study because of multiple factors affecting biliary complications following liver transplantation, and it was important to dispel the myth propagated by most non-transplant biliary surgeons. No laparoscopic HJs have been included in this study. It would, however, be pertinent to analyze the long-term results of laparoscopic $\mathrm{HJ}$ with regard to anastomotic strictures because paradoxically, most laparoscopic surgeons routinely perform a CSHJ during laparoscopic surgeries; however, many of them resort to ISHJ during open surgery.

In conclusion, long-term results, moribidity and mortality associated with CSHJ and ISHJ are similar. However, CSHJ is more economical and quicker, and is associated with a lower bile leak rate than ISHJ based on the analysis of $556 \mathrm{HJs}$, and therefore merits routine use in suitable ducts.

\section{ACKNOWLEDGEMENTS}

The authors gratefully acknowledge Profs. VK Kapoor, Ashok Kumar, Anu Behari, Rajneesh Kumar Singh, Ashok Kumar II, Supriya Sharma, Rahul and Ashish Singh for their contribution to the case material involving the ISHJ group.

\section{CONFLICT OF INTEREST}

No potential conflict of interest relevant to this article was reported.

\section{ORCID}

Rajan Saxena, https://orcid.org/0000-0002-3122-0140

Nalini Kanta Ghosh, https://orcid.org/0000-0003-1213-0235

Saurabh Galodha, https://orcid.org/0000-0002-3966-7190

\section{AUTHOR CONTRIBUTIONS}

Conceptualization: RS. Data curation: All authors. Methodology: RS, NKG. Visualization: RS, NKG. Writing - original draft: RS, NKG. Writing - review \& editing: RS, NKG.

\section{REFERENCES}

1. Kasahara M, Egawa H, Takada Y, Oike F, Sakamoto S, Kiuchi T, et al. Biliary reconstruction in right lobe living-donor liver transplantation: comparison of different techniques in 321 recipients. Ann Surg 2006;243:559-566.

2. Soejima Y, Taketomi A, Yoshizumi T, Uchiyama H, Harada N, Ijichi $\mathrm{H}$, et al. Biliary strictures in living donor liver transplantation: incidence, management, and technical evolution. Liver Transpl 2006;12:979-986.

3. Castaldo ET, Pinson CW, Feurer ID, Wright JK, Gorden DL, Kelly $\mathrm{BS}$, et al. Continuous versus interrupted suture for end-to-end biliary anastomosis during liver transplantation gives equal results. Liver Transpl 2007;13:234-238.

4. Tsui TY, Schlitt HJ, Obed A. Prospective evaluation of biliary reconstruction with duct-to-duct continuous suture in adult live donor liver transplantation. Langenbecks Arch Surg 2011;396:209-215.

5. Dindo D, Demartines N, Clavien PA. Classification of surgical complications: a new proposal with evaluation in a cohort of 6336 patients and results of a survey. Ann Surg 2004;240:205-213.

6. McDonald ML, Farnell MB, Nagorney DM, Ilstrup DM, Kutch JM. Benign biliary strictures: repair and outcome with a contemporary approach. Surgery 1995;118:582-590; discussion 590-591.

7. Antolovic D, Koch M, Galindo L, Wolff S, Music E, Kienle P, et al. Hepaticojejunostomy--analysis of risk factors for postoperative bile leaks and surgical complications. J Gastrointest Surg 2007;11:555-561.

8. Galodha S, Saxena R. Economic and safety implications of continuous versus interrupted suturing method of hepaticojejunostomy: a prospective study. HPB (Oxford) 2016;18(Suppl 1):E495.

9. de Castro SM, Kuhlmann KF, Busch OR, van Delden OM, Laméris JS, van Gulik TM, et al. Incidence and management of biliary leakage after hepaticojejunostomy. J Gastrointest Surg 2005;9:1163-1171; discussion 1171-1173.

10. Asano T, Natsume S, Senda Y, Sano T, Matsuo K, Kodera Y, et al. Incidence and risk factors for anastomotic stenosis of continuous hepaticojejunostomy after pancreaticoduodenectomy. J Hepatobiliary Pancreat Sci 2016;23:628-635.

11. Kadaba RS, Bowers KA, Khorsandi S, Hutchins RR, Abraham AT, Sarker SJ, et al. Complications of biliary-enteric anastomoses. Ann R Coll Surg Engl 2017;99:210-215.

12. Brunner M, Stockheim J, Krautz C, Raptis D, Kersting S, Weber GF, et al. Continuous or interrupted suture technique for hepaticojejunostomy? A national survey. BMC Surg 2018;18:84.

13. Abdel Hamid MAS, Ahmed Rady M, Abd-Elrazik MA. Interrupted versus continuous suturing techniques in hepaticojejunostomy, a retrospective study. J Am Sci 2020;16:23-27. 